\title{
Understanding Saturn's interior from the Cassini Grand Finale gravity measurements
}

\author{
Dongdong Ni \\ State Key Laboratory of Lunar and Planetary Sciences, Macau University of Science and Technology, Macao, PR China \\ e-mail: ddni@must.edu.mo
}

Received 26 April 2020 / Accepted 21 May 2020

\begin{abstract}
Context. Measurements of Saturn's gravity field by Cassini Grand Finale have been acquired with high precision. It has been demonstrated that the even gravitational harmonics $J_{6}-J_{10}$ have larger absolute values than the predictions by typical rigid-body interior models. A four-layer structure model, proposed to interpret Juno's gravity measurements for Jupiter, has been applied to Saturn, but great attention was paid to the depth of zonal flows in order to interpret the large absolute values of $J_{6}-J_{10}$.

Aims. We aim to understand the internal structure and interior composition of Saturn with a similar model for Jupiter. The additional uncertainties in Saturn's structure and composition are investigated in detail, such as rotation periods, atmospheric helium mass fractions, and flow-induced gravity corrections. Also, we investigate the effect of equations of state for hydrogen and helium on the predictions of the core mass and heavy element abundance.

Methods. In the four-layer structure model, we adjusted the heavy element abundances in the outer two envelopes and the mass of the compact core in order to reproduce Saturn's equatorial radius as well as the Cassini Grand Finale gravity measurements corrected by the flow-induced gravity signals. Different four-layer interior models are specified in terms of the rotation period, the atmospheric helium mass fraction, and the flow-induced gravity corrections. Two different ab initio equations of state for hydrogen and helium were used in interior structure calculations. Optimized calculations were then performed to explore Saturn's internal structure and composition.

Results. It is found that the absolute values of $J_{6}-J_{10}$ tend to increase with increasing deep rotation rate and depend on the equations of state adopted in interior calculations. Saturn's deep rotation rate and atmospheric helium mass fraction are important to determine the distribution of helium and heavy elements in the outer envelopes. We also show that the core mass and heavy element abundance in Saturn are dependent upon the deep rotation rate, the atmospheric helium mass fraction, the flow-induced gravity corrections, and the equations of state for hydrogen and helium.
\end{abstract}

Key words. planets and satellites: individual: Saturn - planets and satellites: interiors - planets and satellites: composition

\section{Introduction}

The Cassini spacecraft has measured Saturn's gravitational field to high precision using five of the 22 Grand Finale orbits (Iess et al. 2019). The accuracy of the even harmonics $J_{2}, J_{4}$, and $J_{6}$ has been significantly improved compared with the previous values detected by the flyby missions and by the earlier Cassini orbits (Jacobson et al. 2006). The higher-order harmonics $J_{8}-J_{12}$ were measured through precise Doppler tracking of the Cassini spacecraft. These new gravity data provide excellent observational constraints on Saturn's internal structure. The Cassini Grand Finale gravity measurements reveal larger values for the gravitational harmonics $J_{6}-J_{10}$ with respect to the predictions by typical rigid-body interior models (Iess et al. 2019; Galanti et al. 2019). In order to reconcile the calculated gravitational harmonics with these large values of $J_{6}-J_{10}$, Iess et al. (2019) proposed two different approaches to introduce differential rotation into Saturn's interior models. The first approach is to approximate the wind profile as differential rotation on cylinders and simultaneously optimize interior parameters as well as differential rotation profiles, for which the gravity measurements $J_{2}-J_{10}$ are well reproduced within the error bars. Militzer et al. (2019) further generalized this approach with an accelerated concentric maclaurin spheroid (CMS) method to explore Saturn's interior and rotation. The second approach is to assume the interior shows rigid-body rotation and consider the zonal-flow effect using thermal wind equations, for which the higher-order gravity harmonics $J_{8}$ and $J_{10}$ are well reproduced. In order to interpret all the even gravity harmonics measured by Cassini, Galanti et al. (2019) constructed a wide range of rigid-body interior models and reanalyzed Saturn's flow-induced gravity signal using thermal wind balance. Additionally, Kong et al. (2019) introduced a dynamo region into Saturn's interior and estimated the flowinduced gravity corrections using perturbation approaches. The model interprets all the even zonal gravity harmonics measured by the Cassini Grand Finale mission.

Based on the first approach, the works of Iess et al. (2019) and Militzer et al. (2019) not only interpreted the even gravitational harmonics measured by Cassini but also discussed the interior composition of Saturn in detail. By contrast, the studies using the second approach paid great attention to deducing the depth of zonal flows from the even gravitational harmonics (Galanti et al. 2019; Kong et al. 2019), but still little information is available about Saturn's interior composition. In this study, we aim to understand the internal structure and interior composition of Saturn using a similar model for Jupiter, in order to judge whether a consistent picture works for both planets. The interiors of Jupiter and Saturn were compared by Guillot (1999) within 
the framework of the three-layer assumption, and the effects of shape and rotation on Saturn's interior were also explored using three-layer interior models (Helled \& Guillot 2013). Recently, we constructed a wide range of four-layer interior models to investigate Jupiter's internal structure and composition from Juno gravity measurements ( $\mathrm{Ni}$ 2019). This motivates consideration of how the four-layer model works for the understanding of Saturn's interior. Furthermore, it is of great interest to discern whether there are common features with respect to the predictions of Militzer et al. (2019). In Sect. 2, we describe four-layer structure models specified for Saturn. We calculate the gravitational harmonics in terms of the fifth-order theory of figures. We use the newly detected data from the Cassini Grand Finale, corrected by the dynamical contributions of Galanti et al. (2019) and Kong et al. (2019), to constrain the internal structure of Saturn. In Sect. 3, the practical aspects of optimization calculations are presented and three cases of four-layer interior models are specified in terms of Saturn's rotation period and flow-induced gravity signals. Section 4 presents the interior model predictions of gravitational harmonics, helium mass fractions, and heavy element amounts. The effects of rotation periods and atmospheric helium mass fractions on the internal structure of Saturn are discussed in detail, together with the sensitivity of the predictions to the equation of state (EOS) adopted in the interior models. Finally, the main results of this work are summarized in Sect. 5.

\section{Four-layer interior structure models for Saturn}

We consider Saturn's interior structure to be similar to the four-layer structure of Jupiter. Following the previous work of $\mathrm{Ni}$ (2019), the radial structure of Saturn is assumed to consist of four layers: (1) a homogeneous molecular hydrogen atmosphere in which helium is depleted, (2) a homogeneous metallic hydrogen envelope in which helium is enriched, (3) a dilute core region with enriched heavy elements, and (4) an isothermal central compact core of rock. The outer two layers should be divided by a nonadiabatic region of hydrogen-helium immiscibility, but there are still uncertainties in the $\mathrm{H} / \mathrm{He}$ phase separation (Lorenzen et al. 2011; Morales et al. 2013; Schöttler \& Redmer 2018), and such a nonadiabatic behavior has rather little influence on the gravitational harmonics with respect to the uncertainties discussed in this work (Galanti et al. 2019; Nettelmann et al. 2015). Therefore, the alternative procedure is to ignore the nonadiabatic region in Saturn's internal structure and to consider the nonadiabatic effect on Saturn's thermodynamical properties. In this case, a temperature jump $\Delta T_{1-2}$ is introduced across the interface between these two layers, since the nonadiabatic region reduces the heat transport from Saturn's deeper interior (Hubbard \& Militzer 2016; Nettelmann et al. 2015). The mass fraction of helium and heavy elements in the outer three layers are denoted by $\left(Y_{1}, Z_{1}\right),\left(Y_{2}, Z_{2}\right)$, and $\left(Y_{3}\right.$, $Z_{3}$ ), respectively. Based on current knowledge of gas giant planets, there are three requirements on the element abundances in Saturn's interior. First, the upper molecular atmosphere has a smaller helium mass fraction than the lower metallic envelope $\left(Y_{1}<Y_{2}\right)$ owing to helium rainout. Second, the dilute core region is a diffusive region in which the heavy elements are enriched compared to the exterior envelope region. This is attributed to the heavy elements dissolved from the central rocky core into the metallic hydrogen and expanded outward through a portion of the planet (Wahl et al. 2017). Therefore, there is an increase in the heavy element abundance for the dilute core region, $\Delta Z_{2-3}=Z_{3}-Z_{2}>0$. Given the enriched heavy elements of ices and rocks, we introduced one free parameter $f_{\text {rock }}$ for the

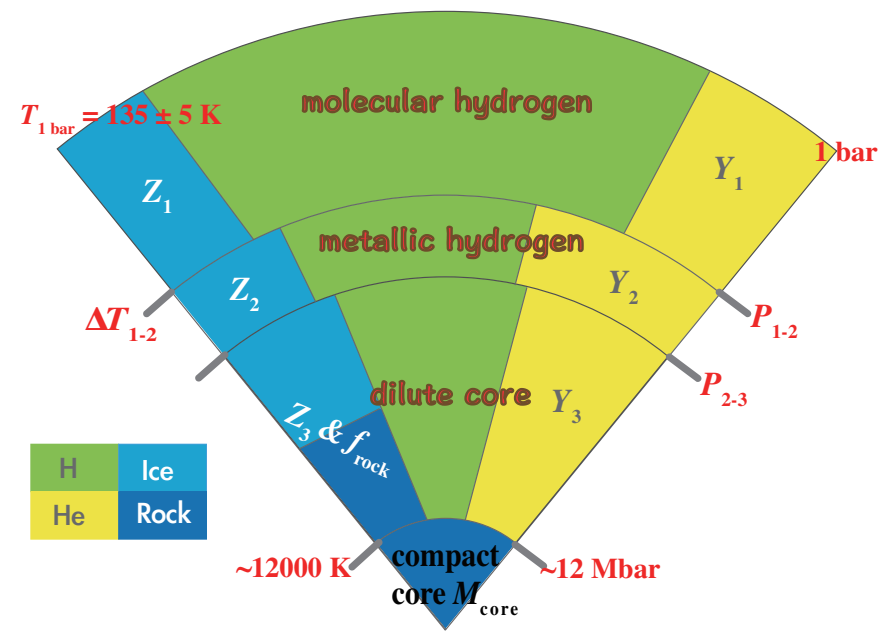

Fig. 1. Schematic four-layer structure of Saturn. There is a temperature jump $\Delta T_{1-2}$ between layers 1 and 2 owing to a thin helium rain layer, and the interface between layers 2 and 3 is described with the pressure $P_{2-3}$.

dilute core region, which is defined as the rock fraction in heavy elements $f_{\text {rock }}=Z_{\text {rock }} / Z_{3}$. Third, the mean helium mass fraction in Saturn $Y /(1-Z)$ should be equal to its value in the protosolar nebula $Y_{\text {proto }} /\left(1-Z_{\text {proto }}\right)=0.277 \pm 0.006$ (Serenelli \& Basu 2010; Guillot et al. 2018; Ni 2019). The correlation between $Y_{1}, Y_{2}$, and $Y_{3}$ can be found in the appendix of $\mathrm{Ni}$ (2019). Figure 1 illustrates a schematic diagram of Saturn's four-layer structure. The interface between layers 1 and 2 is characterized by the pressure $P_{1-2}$ and the interface between layers 2 and 3 is described with the pressure $P_{2-3}\left(P_{2-3}>P_{1-2}\right)$.

The CMS method and theory of figures (ToF) have been used to calculate the self-consistent shape and gravity field of rotating liquid planets (Hubbard 2013; Hubbard \& Militzer 2016; Debras \& Chabrier 2018; Nettelmann 2017; Ni 2018; Guillot et al. 2018). The CMS confers an advantage in that it is mathematically simple and shows good accuracy. Its disadvantage is that it requires quite a large amount of computation despite the accelerated CMS method (Militzer et al. 2019). By contrast, the ToF is famous for its high numerical efficiency (Zharkov \& Trubitsyn 1978), but a high-order approximation is required for the ToF in order to match the error bars of the Cassini Grand Finale gravity measurements. In view of a large ensemble of possible four-layer configurations and a number of iterations necessary to fit the available measurements, we employ the ToF to fifth order (ToF5) to calculate Saturn's level surface (Zharkov \& Trubitsyn 1975; Ni 2018):

$r(s, \theta)=s\left[1+\sum_{i=0}^{5} s_{2 i}(s) P_{2 i}(\cos \theta)\right]$.

The ToF5 accuracy is examined in Appendix A by comparison with the accelerated CMS results. It is found that its accuracy is comparable with the accelerated CMS method and it is able to interpret the Cassini Grand Finale gravity measurements. Using the computed figure functions $s_{2 i}(s)$ and abbreviating the level surface as $r=s\left(1+\sum\right)$, one can evaluate the even zonal harmonics as (Guillot et al. 2018; Ni 2019)

$$
\begin{aligned}
J_{2 i}= & -\frac{3}{2}\left(\frac{R_{\mathrm{p}}}{R_{\mathrm{eq}}}\right)^{2 i} \int_{-1}^{1} \mathrm{~d} \cos \theta P_{2 i}(\cos \theta) \int_{0}^{1} \mathrm{~d} x \\
& \times x^{2 i+2}\left(1+\sum\right)^{2 i+2} \eta(x)\left(\frac{\mathrm{d} r}{\mathrm{~d} s}\right),
\end{aligned}
$$


Table 1. Saturn's rigid-rotation gravity harmonics $J_{2 i}^{\text {rigid }}$, which are derived by subtracting the flow-induced contributions $\Delta J_{2 i}$ (Galanti et al. 2019; Kong et al. 2019) from the Cassini Grand Finale measurements $J_{2 i}^{\text {Cassini }}$ (Iess et al. 2019).

\begin{tabular}{|c|c|c|c|c|c|}
\hline \multirow{2}{*}{$\begin{array}{l}\text { Parameter } \\
J_{2} \times 10^{6}\end{array}$} & \multirow{2}{*}{$\begin{array}{c}J_{2 i}^{\text {Cassini }} \\
\text { Iess et al. (2019) } \\
16324.108 \pm 0.028\end{array}$} & \multicolumn{2}{|c|}{$\begin{array}{c}\Delta J_{2 i} \\
\text { Galanti et al. (2019) } \\
J_{2 i}^{\text {rigid }} \\
\end{array}$} & \multicolumn{2}{|c|}{$\begin{array}{cc}\Delta J_{2 i} & J_{2 i}^{\text {rigid }} \\
\text { Kong et al. (2019) }\end{array}$} \\
\hline & & 50.540 & $16273.568 \pm 0.028$ & -3.855 & $16327.963 \pm 0.02$ \\
\hline$J_{4} \times 10^{6}$ & $-939.169 \pm 0.037$ & 7.588 & $-946.757 \pm 0.037$ & 3.032 & $-942.201 \pm 0.037$ \\
\hline$J_{6} \times 10^{6}$ & $86.874 \pm 0.087$ & 4.093 & $82.781 \pm 0.087$ & 2.187 & $84.687 \pm 0.087$ \\
\hline$J_{8} \times 10^{6}$ & $-14.745 \pm 0.205$ & -5.506 & $-9.239 \pm 0.205$ & -5.023 & $-9.722 \pm 0.205$ \\
\hline$J_{10} \times 10^{6}$ & $4.720 \pm 0.420$ & 3.660 & $1.060 \pm 0.420$ & 3.399 & $1.321 \pm 0.420$ \\
\hline
\end{tabular}

Notes. The Cassini Grand Finale measurements of Iess et al. (2019) are normalized to the reference radius $60268 \mathrm{~km}$ instead of $60330 \mathrm{~km}$.

where $R_{\mathrm{eq}}$ is the equatorial radius of the planet, $R_{\mathrm{p}}$ is the mean radius of the planet, $x$ is the scaled mean radius, $x=s / R_{\mathrm{p}}, \eta(x)$ is the normalized density distribution, and $(d r / d s)$ is the derivative of $r$ with respect to $s$ obtained from Eq. (1).

The even zonal gravitational harmonics measured by the Cassini Grand Finale are contributed from a rigidly rotating planet $J_{2 i}^{\text {rigid }}$ and differentially rotating zonal flows $\Delta J_{2 i}$. The rigid-rotation contribution is obtained by subtracting the flowinduced contribution from the Cassini Grand Finale gravity measurements, $J_{2 i}^{\text {rigid }}=J_{2 i}^{\text {Cassini }}-\Delta J_{2 i}$. Very recently, Galanti et al. (2019) explored Saturn's flow-induced gravity signals using thermal wind balance and Kong et al. (2019) estimated Saturn's flow-induced gravity corrections using perturbation approaches. The flow-induced gravitational harmonics $\Delta J_{2 i}$ and the resulting rigid-rotation contribution $J_{2 i}^{\text {rigid }}$ are shown in Table 1 . We note that the rigid-rotation gravity harmonics are used for reference in the following optimization calculations.

\section{Practical aspects}

One important input quantity in the interior models of giant planets is the EOS (Miguel et al. 2016; Saumon \& Guillot 2004), that is, the density as a function of temperature, pressure, and composition. For hydrogen and helium, we use two ab initio EOSs in this work: the REOS3b for which the zero point of the specific internal energy in the REOS.3 tables of Becker et al. (2014) is changed to coincide in the ideal gas regime with the $\mathrm{SCvH}$ EOS, and the $\mathrm{MH} 13+\mathrm{SCvH}$ which is extracted from the tables of Militzer \& Hubbard (2013, MH13) for hydrogenhelium mixtures using the $\mathrm{SCvH}$ EOS. For a minor amount of heavy elements, we use the EOS of silicates dry sand in SESAME for rocks and the SESAME EOS 7154 of water for ices (Lyon \& Johnson 1992). For mixtures of hydrogen, helium, and heavy elements, the EOS is constructed using the separate EOSs for pure species in terms of the additive volume rule.

The numerical model is established based on the CEPAM package (Guillot \& Morel 1995). The outer boundary condition at 1 bar is set as $T=135 \pm 5 \mathrm{~K}$ based on Voyager measurements (Lindal 1992). The heavy element abundances $Z_{1}$ and $Z_{2}$ as well as the compact core mass $M_{\text {core }}$ are adjusted to reproduce Saturn's equatorial radius $R_{\mathrm{eq}}$ and even low-order gravitational harmonics $J_{2}, J_{4}, J_{6}$. The other parameters are poorly constrained at present, such as the pressure $P_{1-2}$ for the $\mathrm{H} / \mathrm{He}$ phase transition, the temperature jump $\Delta T_{1-2}$ accounting for the nonadiabatic effect of helium rainout, and the size and composition

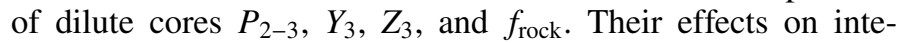
rior models have been investigated in previous works (Miguel et al. 2016; Ni 2019) and are beyond the scope of this work; in our calculations, they are adopted beforehand based on the current knowledge of Saturn's interior. The pressure for the $\mathrm{H} / \mathrm{He}$ phase transition is fixed at $P_{1-2}=1.0$ Mbar (Morales et al. 2013; Lorenzen et al. 2011) and the temperature jump $\Delta T_{1-2}$ is uniformly varied from 0 to $1000 \mathrm{~K}$. We note that increasing the $P_{1-2}$ value causes fewer heavy elements in Saturn's core and hotter deep interiors allow a larger amount of heavy elements in Saturn's interior (Miguel et al. 2016; Ni 2019). The pressure $P_{2-3}$ of the dilute core region is uniformly varied from 2 to $8 \mathrm{Mbar}$ and some interior models without dilute cores are considered as well to cover all possible configurations in Saturn's interior. As demonstrated in Ni (2019), the compositions in the deeper layer, $Y_{3}$ and $Z_{3}$, are chosen randomly within a broad range of $\Delta Z_{2-3}=0-0.2$ and $\Delta Y_{2-3} \leq 0.2$ under the requirement that the heavy elements become enriched in the dilute core region and the density steadily increases from the metallic envelope to the dilute core region. The rock fraction in the dilute core region is fixed at a median value of $f_{\text {rock }}=0.50$. We note that increasing the rock fraction $f_{\text {rock }}$ tends to lower the amount of heavy elements in Saturn's interior (Ni 2019).

In contrast to Jupiter's interior models, there are two additional issues in modeling Saturn's interior. One is the uncertainty on the planet's deep rotation rate due to the near-perfect alignment of the magnetic field dipole with the rotation axis (Anderson \& Schubert 2007). Galanti et al. (2019) explored Saturn's deep atmospheric flows with two rotation periods $10 \mathrm{~h}$ $32 \mathrm{~min} 45 \mathrm{~s}$ (Helled et al. 2015, fast) and $10 \mathrm{~h} 39 \mathrm{~min} 22 \mathrm{~s}$ (Smith et al. 1982, slow) for deep rigid-body rotation. Kong et al. (2019) investigated Saturn's zonal circulation of the molecular layer with the bulk rotation period of $10 \mathrm{~h} 33 \mathrm{~min} 38 \mathrm{~s}$ (Mankovich et al. 2019, medium). Combining the flow-induced gravity corrections with different rotation periods, we consider three cases in this work. Cases (I) and (II) correspond to the flow-induced gravity corrections of Galanti et al. (2019) with the fast and slow rotation rates, respectively, and Case (III) corresponds to the flow-induced gravity corrections of Kong et al. (2019) with the medium rotation rate. The other issue in modeling Saturn's interior is the uncertainty of the atmospheric helium mass fraction, because no entry probe has been achieved for Saturn's atmosphere. The reexamination of Voyager measurements suggested a helium mass fraction in Saturn's atmosphere of $0.18-0.25$ relative to total hydrogen and helium (Conrath \& Gautier 2000). The evolutionary models of Hubbard et al. (1999) constrained the atmospheric helium mass fraction between 0.11 and 0.21 relative to total helium and hydrogen. In this work, we uniformly vary the atmospheric helium mass fraction $Y_{1} /\left(X_{1}+Y_{1}\right)$ from 0.130 to 0.230 in order to discern its effect on Saturn's interior. Given $Y_{1}$ and $Y_{3}$, the helium mass fraction in the middle envelope $Y_{2}$ is adjusted to make sure that the total helium to hydrogen ratio 

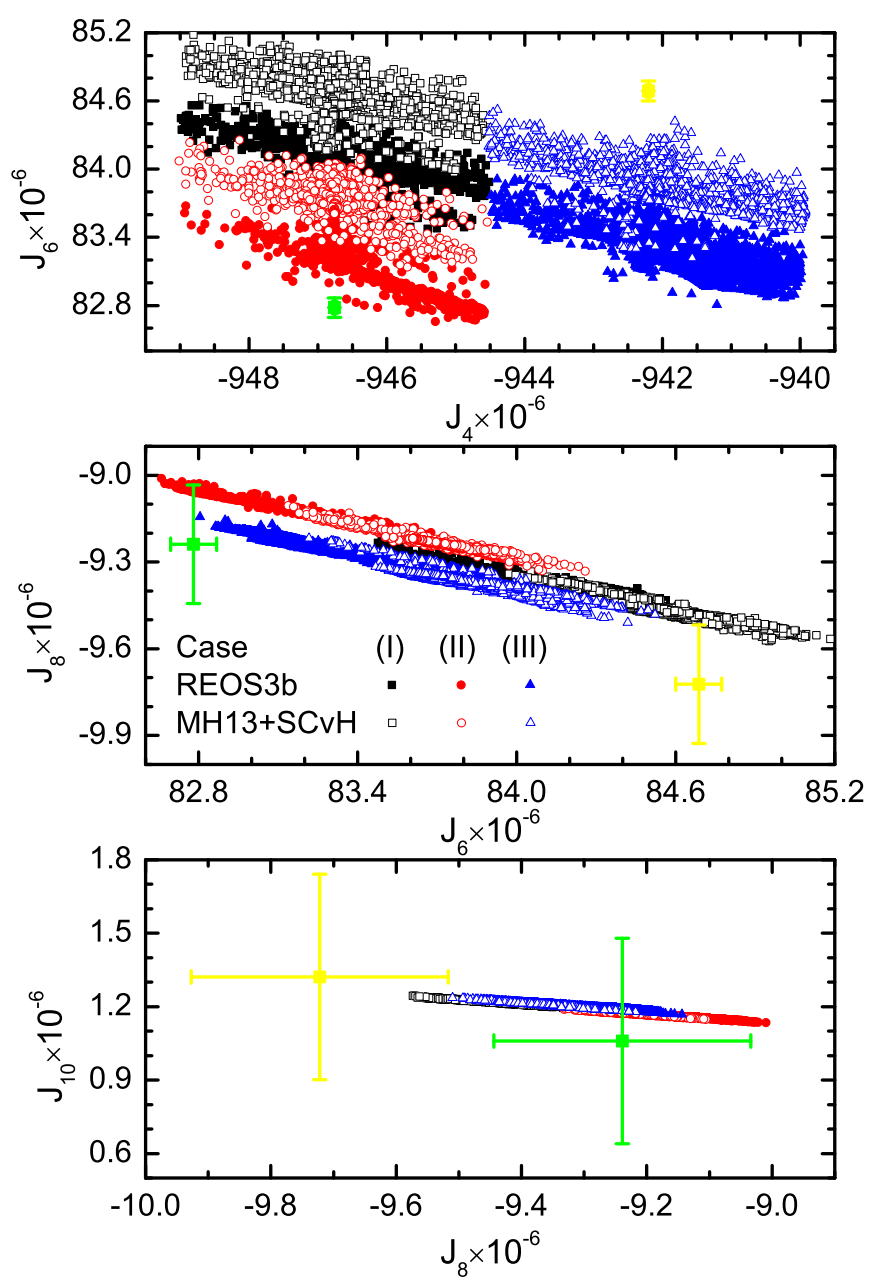

Fig. 2. Gravity harmonics for Saturn obtained with various models in the $J_{i}-J_{i+2}$ planes. The model solutions are shown for Cases (I-III) with the different EOSs used in the interior models: REOS3b (solid points) and $\mathrm{MH} 13+\mathrm{SCvH}$ (open points). The rigid-rotation gravity harmonics $J_{2 i}^{\text {rigid }}$ corrected by the dynamical contributions of Galanti et al. (2019) and Kong et al. (2019) are displayed as green and yellow solid squares, respectively, with $1 \sigma$ error bars.

in Saturn $Y /(1-Z)$ is equal to its value in the protosolar nebula $Y_{\text {proto }} /\left(1-Z_{\text {proto }}\right)=0.277 \pm 0.006$ (Serenelli \& Basu 2010). Here, great attention is paid to the effect of rotation periods and atmospheric helium abundances on Saturn's interior models.

\section{Model results}

\subsection{Saturn's even zonal gravity harmonics}

In view of the high precision of Cassini Grand Finale gravity measurements and the dynamical uncertainties of differentially rotating zonal flows, we allow the model solutions to deviate from the measured data within $\sim 30 \sigma$ to cover a wide range of interior configurations. Figure 2 shows the even gravity harmonics obtained with various models compared with the rigid-rotation gravity harmonics $J_{2 i}^{\text {rigid }}$. Green and yellow solid squares stand for the rigid-rotation gravity harmonics $J_{2 i}^{\text {rigid }}$ corrected by the dynamical contributions of Galanti et al. (2019) and Kong et al. (2019), respectively. One can see that the $J_{2 i}^{\text {rigid }}$ values corrected by Galanti et al. (2019) exhibit lower absolute values for the high-order harmonics $J_{6}$ to $J_{10}$ than those corrected by

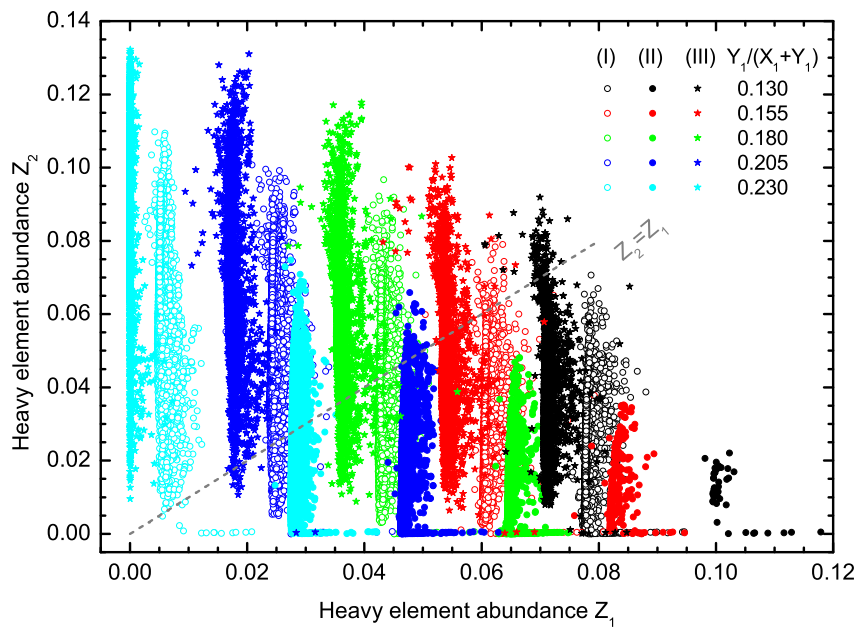

Fig. 3. Heavy element abundances in the molecular and metallic envelopes obtained with REOS3b for Cases (I) (open circles), Case (II) (solid circles), and Case (III) (solid stars). The optimization solutions are displayed in different colors according to different helium depletions $Y_{1} /\left(X_{1}+Y_{1}\right)$.

Kong et al. (2019). Also shown is the uncertainty $\sigma$ associated with Cassini Grand Finale measurements. The dynamical contributions to Saturn's zonal gravity harmonics are larger than the very small measurement uncertainty $\sigma$, as shown in Table 1 . In this case, a wide range of possible internal flows and dynamical considerations significantly increase the effective uncertainty of the rigid-rotation harmonics $J_{2 i}^{\text {rigid }}$. Kaspi et al. (2017) explored the effective uncertainty of Jupiter's static gravity harmonics by investigating the effect of differential rotation. The effective uncertainty on the static $J_{2}-J_{8}$ is indeed greater than the Juno measurement uncertainty by orders of magnitude. The effect of differential rotation is expected to be greater for Saturn than for Jupiter. Future work should consider the effective uncertainty on Saturn's rigid-rotation gravity harmonics in the fitting procedure.

As can be seen in Fig. 2, the models yield $J_{4}$ closely around the fitted $J_{4}^{\text {rigid }}$ values and the calculated harmonics $J_{6}$ to $J_{10}$ fall in between the $J_{2 i}^{\text {rigid }}$ values corrected by Galanti et al. (2019) and by Kong et al. (2019). These harmonics show good agreement with the rigid-rotation gravity harmonics within the effective uncertainties. For Cases (I) and (II) respectively with the fast and slow rotation rates, the former seems to yield larger absolute values for the high-order gravity harmonics $J_{6}$ to $J_{10}$. This is consistent with the results of Galanti et al. (2019). Moreover, $\mathrm{MH} 13+\mathrm{SCvH}$ also yields larger absolute values for $J_{6}$ to $J_{10}$ than REOS3b. This can be explained as MH13+SCvH exhibiting higher densities for all the temperatures than REOS3b (Miguel et al. 2016). The high-order gravity harmonics are sensitive to the density in the outer part of a planet (Guillot 2005) and their absolute values generally increase with the local density (Ni 2019).

\subsection{Saturn's element abundance in the envelopes}

With the Cassini Grand Finale gravity measurements, it would be interesting to explore Saturn's element abundances in the outer envelopes. In the four-layer structure model, the heavy element abundances in the outer two envelopes $\left(Z_{1}\right.$ and $\left.Z_{2}\right)$ and the mass of the central compact core $\left(M_{\text {core }}\right)$ are adjusted to match the observational constraints. In this section, we take the 

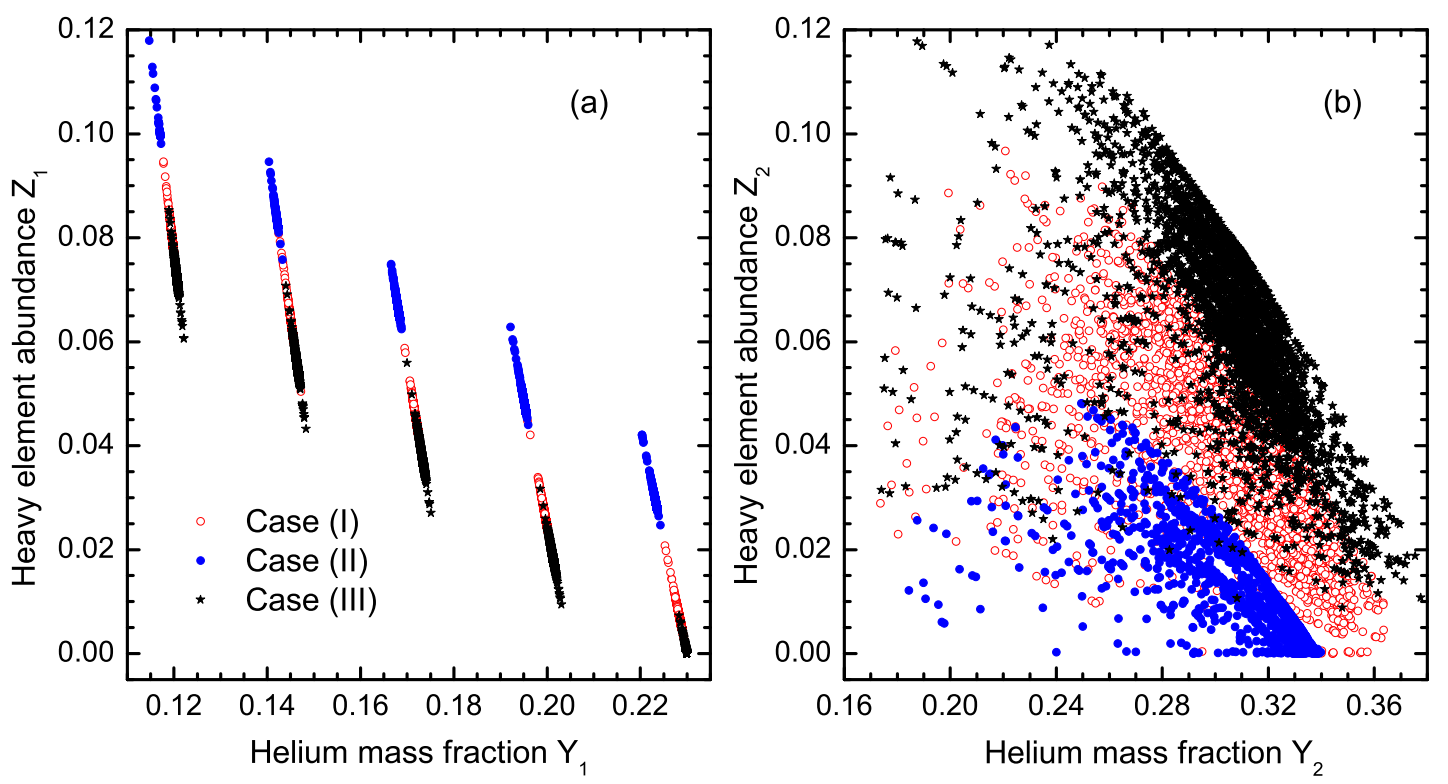

Fig. 4. Saturn's element abundances in the outer envelopes obtained with REOS3b for Case (I) (red open circles), Case (II) (blue solid circles), and Case (III) (black solid stars). Panel a: trade-off between heavy element and helium mass fractions in the molecular envelope. Panel $b$ : trade-off between heavy element and helium mass fractions in the metallic envelope. We note that panel $b$ applies to $Y_{1} /\left(X_{1}+Y_{1}\right)=0.180$, but would remain very similar for the other $Y_{1} /\left(X_{1}+Y_{1}\right)$ values.

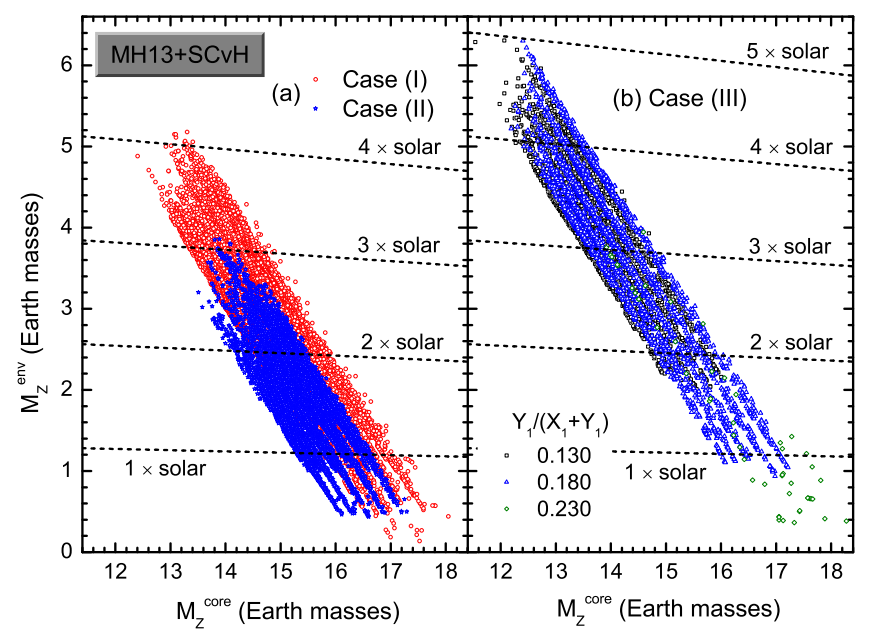

Fig. 5. Mass of heavy elements in the core and in the envelopes predicted with $\mathrm{MH} 13+\mathrm{SCvH}$. The heavy element abundances in the envelopes are indicated by dashed lines in units of the solar abundance of heavy elements. (a) The results of Cases (I) and (II) are displayed in red circles and in blue stars, respectively, reflecting the effect of the different rotation period. $(b)$ The results of Case (III) are displayed according to different helium depletions $Y_{1} /\left(X_{1}+Y_{1}\right)$ as specified in the legend.

optimization slolutions using REOS3b for example. The resulting heavy element abundances in the outer two envelopes are shown in Fig. 3 for Cases (I-III), which are derived with different $Y_{1} /\left(X_{1}+Y_{1}\right)$ values. With less helium depletion in the molecular envelope corresponding to an increase of $Y_{1} /\left(X_{1}+Y_{1}\right)$, the $Z_{1}$ value is decreased but the $Z_{2}$ value is allowed to vary in a wider range. Taking Case (I) for example, the $Z_{1}$ value is decreased from 5 to 0.5 times solar fraction while the $Z_{2}$ variation is changed from $0-5$ to $0-7$ times solar fraction. The increased flexibility of $Z_{2}$ can be understood because the dilute core region is allowed to vary in both size $\left(P_{2-3}\right)$ and composition $\left(Z_{3}\right.$ and $\left.Y_{3}\right)$ while the mean helium-to-hydrogen ratio is fixed for the molecular envelope. In addition, comparing Case (I) with Case (II), we find that the solutions with the slow rotation rate are confined to a narrow area in the figure, suggesting more heavy-element enrichment in the molecular envelope. By contrast, the solutions of Case (III) tend to the area of $Z_{2}>Z_{1}$ in particular for less helium depletion in the molecular envelope.

The correlations between the abundances of helium and heavy elements are illustrated in Fig. 4 for the outer two envelopes. Figure $4 \mathrm{a}$ shows the heavy-element abundance $Z_{1}$ as a function of the helium mass fraction $Y_{1}$ in the molecular envelope. In practical calculations, the $Y_{1} /\left(X_{1}+Y_{1}\right)$ value is uniformly adopted beforehand and the $Z_{1}$ value is determined from the observational constraints. As can been seen, there exists a good correlation between $Y_{1}$ and $Z_{1}$ for all three cases and for various amounts of helium depletion. The amount of heavy elements in the molecular envelope decreases from Case (II) with the slow rotation rate, to Case (I) with the fast rotation rate, and then again to Case (III) with the flow-induced gravity correction of Kong et al. (2019). In Fig. 4b, we plot the heavy element abundance $Z_{2}$ versus the helium mass fraction $Y_{2}$ in the metallic envelope. The $Y_{2}$ value is determined by the requirement that the total helium-to-hydrogen ratio in Saturn be equal to its value in the protosolar nebula, and the $Z_{2}$ value is adjusted in terms of the observational constraints. We note that only the solutions with $Y_{1} /\left(X_{1}+Y_{1}\right)=0.180$ are displayed for the sake of clarity and the solutions with the other $Y_{1} /\left(X_{1}+Y_{1}\right)$ values show very similar behaviors. One can identify a tendency toward the smaller $Z_{2}$ value with increasing $Y_{2}$ value, although the solutions have a large spread of available space. The solutions of Case (II), with the slow rotation rate, tend to smaller $Z_{2}$ values in the metallic envelope, while most solutions of Case (III) yield larger $Z_{2}$ values. This is in stark contrast to the situation shown in Fig. 4a. Such contradicting behavior brings in small differences of the heavy element mass in the envelopes for all three cases; this is further discussed below. 


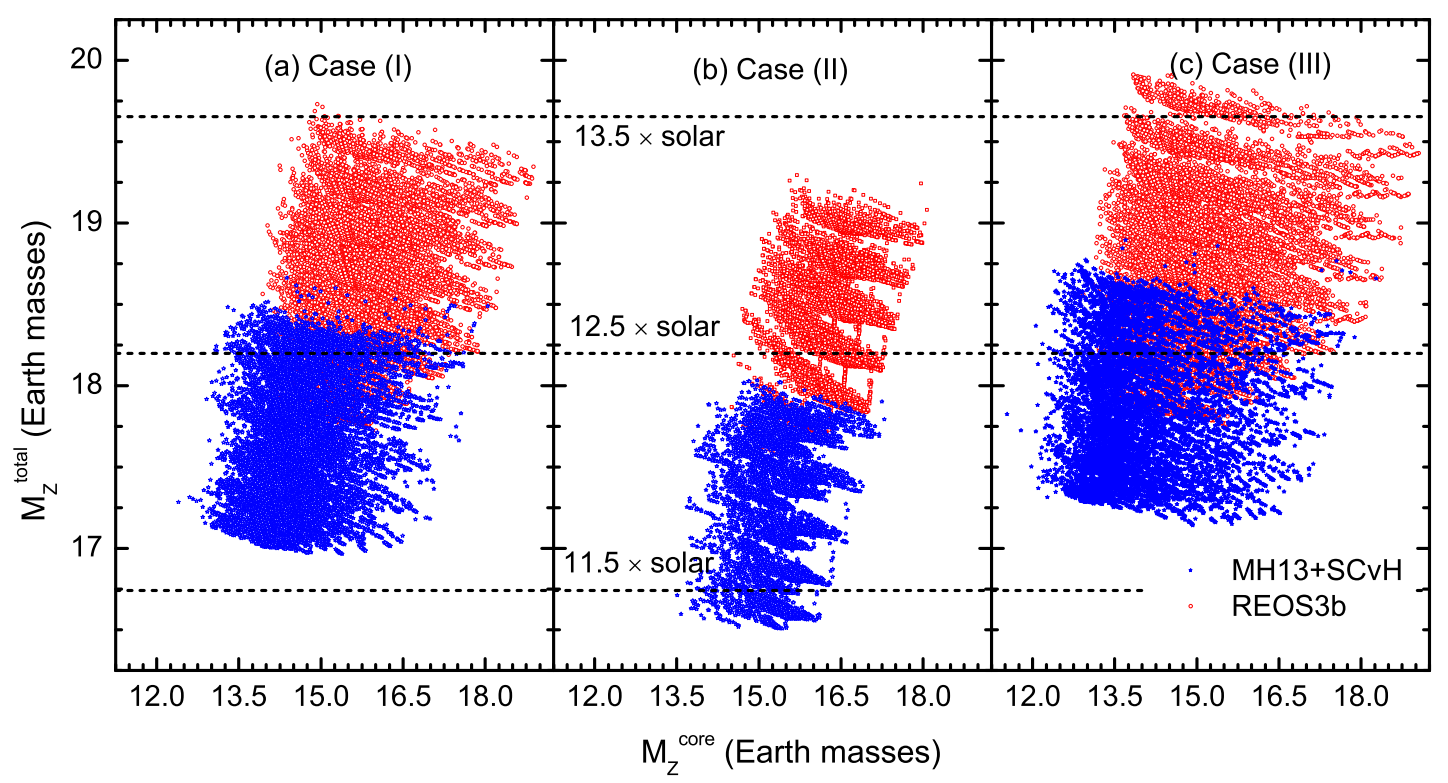

Fig. 6. Mass of the heavy elements in the core and the total heavy element mass in Saturn predicted with different EOSs for hydrogen and helium: $\mathrm{MH} 13+\mathrm{SCvH}$ (blue stars) and REOS3b (red circles). Horizontal lines denote the global mass fraction of heavy elements in units of the solar abundance of heavy elements.

\subsection{Saturn's core and heavy element mass}

The interior models of Saturn are affected by the uncertainties in the zonal flows, rotation periods, atmospheric helium mass fractions, and EOSs. Figure 5 shows the comparison of the heavy-element masses in the core and in the envelopes derived using $\mathrm{MH} 13+\mathrm{SCvH}$ for Cases (I-III). In Fig. 5a, we plot the solutions of Cases (I) and (II) to discern the effect of rotation periods on the model predictions. As can be seen, decreasing the rotation rate causes the solutions to be confined to a narrow area of larger $M_{Z}^{\text {core }}$ and smaller $M_{Z}^{\text {env }}$. In Fig. 5b, we plot the solutions of Case (III) according to different $Y_{1} /\left(X_{1}+Y_{1}\right)$ values to discern the effect of atmospheric helium mass fractions. It seems that less helium depletion in the molecular envelope tends to allow a larger core mass $M_{Z}^{\text {core }}$ and a smaller amount of heavy elements in the envelopes $M_{Z}^{\text {env }}$. Militzer et al. (2019) investigated the effects of rotation periods and core radii using seven sets of interior models with five different rotation periods and three different core radii. As the rotation period is increased, the model solutions move toward an area of larger $M_{Z}^{\text {core }}$ and smaller $M_{Z}^{\text {env }}$ and meanwhile the helium mass fraction in the molecular envelope becomes enhanced with a smaller amount of helium rainout (Militzer et al. 2019). Our results presented here are consistent with the predictions of these latter authors in spite of the different four-layer structure assumptions regarding Saturn's interior. In addition, the heavy-element masses in the core and in the envelopes have some dependence on the flow-induced gravity corrections used to deduce the rigid-rotation gravity harmonics $J_{2 i}^{\text {rigid }}$. As can be seen from panels a and b, the $M_{Z}^{\text {core }}$ values derived in Case (III) reach core masses $1 M_{\oplus}$ smaller than the results of Case (I) and the largest $M_{Z}^{\text {env }}$ value of Case (III) is $1 M_{\oplus}$ larger than the value of Case (I). On the whole, results derived with $\mathrm{MH} 13+\mathrm{SCvH}$ reveal a heavy-element mass in the core of 12-18 $M_{\oplus}$ and a heavy-element mass in the envelopes of up to five times the solar fraction. These results agree with the MH13 predictions of 15-18 $M_{\oplus}$ and 1.2-4.0 times the solar fraction suggested by Militzer et al. (2019). We note that the predictions of Militzer et al. (2019) are dependent on core size. If smaller fractional core radii were taken into account, smaller core masses would be achieved together with a higher heavy-element abundance in the envelopes, leading to better agreement between the two.

Figure 6 shows the sensitivity of Saturn's heavy elements to the EOSs for Cases (I-III). As can be seen, the interior model predictions exhibit some dependence on the adopted EOSs. However, this dependence is less considerable than the corresponding dependence found for Jupiter (Ni 2019). The REOS3b solutions seem to allow a larger amount of heavy elements in Saturn's core and in Saturn as a whole with respect to the $\mathrm{MH} 13+\mathrm{SCvH}$ solutions. This feature is also found in the analogous interior models for Jupiter (Ni 2019). For MH13+SCvH, the heavy element mass in the core $M_{Z}^{\text {core }}$ spans over the range of $12-18 M_{\oplus}$ and the global heavy element abundance $Z_{\text {global }}$ is estimated as 11.5-13.0 times the solar fraction. For REOS3b, $M_{Z}^{\text {core }}$ spans over a range of $13-19 M_{\oplus}$ and $Z_{\text {global }}$ is as high as 12.0-13.5 times the solar fraction. In contrast with the interior model predictions for Jupiter $\left(\mathrm{MH} 13+\mathrm{SCvH}\right.$ : 6.5-27.0 $M_{\oplus}$ and five to six times solar fraction, REOS3b: $7-32 M_{\oplus}$ and roughly seven times solar fraction), the four-layer structure models of Saturn yield a narrower range for the core mass but a higher value for the global heavy element abundance. One can also see that, out of all three cases, the solutions of Case (III) show the greatest range for the core mass and the largest amount of heavy elements in Saturn's interior. The effect of the flow-induced gravity signals is even comparable with that of the rotation period. Furthermore, it should be pointed out that the interior temperature in Saturn could be hotter than what we consider here owing to the nonadiabatic structure with compositional gradients in the deep interior (Vazan et al. 2016). This increased temperature would lead to a larger amount of heavy elements in Saturn's interior (Ni 2019).

\section{Summary}

In order to discern whether Jupiter and Saturn share similar internal structure, the four-layer interior model, which was developed for Jupiter to interpret Juno gravity measurements, has been used 
to understand the internal structure and composition of Saturn from the Cassini Grand Finale gravity measurements. Particular attention is paid to the additional uncertainties for Saturn, such as the deep rotation periods, the atmospheric helium mass fraction, and the flow-induced gravity corrections. Based on current knowledge of Saturn, three rotation periods of Saturn's deep interior are considered and two sources of the flow-induced gravity corrections are adopted. Various helium depletions in the molecular envelope are assumed in the four-layer interior models. Also, two ab initio EOSs for hydrogen and helium (REOS3b and $\mathrm{MH} 13+\mathrm{SCvH})$ are used in interior calculations based on the work of Miguel et al. (2016).

The rigid-rotation gravity harmonics are calculated using two-dimensional integrals with the level surface derived from the ToF5 method. Considering two possible dynamical contributions of Galanti et al. (2019) and Kong et al. (2019), the effective uncertainty of the rigid-rotation gravity harmonics is remarkably increased with respect to the error bar of the Cassini Grand Finale measurements. The four-layer models accurately reproduce the even rigid rotation $J_{2}-J_{10}$ within the effective uncertainty. The high-order gravity harmonics $J_{6}-J_{10}$ are found to have some dependence on the rotation period and the EOS. As shown in Fig. 2, the short-period model yields the larger absolute values of $J_{6}-J_{10}$, and $\mathrm{MH} 13+\mathrm{SCvH}$ yields larger absolute values than REOS3b. Saturn's deep rotation period and atmospheric helium mass fraction affect the distribution of helium and heavy elements in the outer two envelopes. Increasing the deep rotation rate or reducing helium depletion in the molecular envelope lead to a smaller amount of heavy elements in the molecular envelope and allow a larger heavy element abundance in the metallic envelope. Also, there are correlations between the mass fractions of helium and heavy elements in the molecular and metallic envelopes. The heavy element abundances tend to decrease with increasing helium mass fraction, as shown in Fig. 4.

The core mass and heavy element abundance in Saturn's interior depend on the deep rotation rate, the atmospheric helium mass fraction, the flow-induced gravity corrections, and the EOSs for hydrogen and helium. In our baseline simulations, decreasing the rotation rate causes the solutions of $M_{Z}^{\text {core }}$ and $M_{Z}^{\text {env }}$ to be confined to a narrow area, and decreasing helium depletion in the molecular envelope leads to larger $M_{Z}^{\text {core }}$ and smaller $M_{Z}^{\text {env }}$. The flow-induced gravity corrections of Galanti et al. (2019) and Kong et al. (2019) also result in different solutions of $M_{Z}^{\text {core }}$ and $M_{Z}^{\text {env }}$, with a difference of up to $1 M_{\oplus}$ in $M_{Z}^{\text {core }}$ and $1 M_{\oplus}$ in $M_{Z}^{\text {env }}$. The models using $\mathrm{MH} 13+\mathrm{SCvH}$ yield $M_{Z}^{\text {core }}$ of $12-18 M_{\oplus}$ and a global heavy element abundance $Z_{\text {global }}$ of 11.5-13.0 times the solar fraction. The models using REOS3b yield $M_{Z}^{\text {core }}$ of $13-19 M_{\oplus}$ and $Z_{\text {global }}$ of 12.0-13.5 times the solar fraction. By contrast, the analogous interior models for Jupiter predict a wider range of $M_{Z}^{\text {core }}\left(6.5-32 M_{\oplus}\right)$ but a lower value of $Z_{\text {global }}(5-7$ times the solar fraction), together with a stronger dependence on the ab initio EOSs for hydrogen and helium (Ni 2019). New information on Saturn's deep rotation period, atmospheric helium mass fraction, and flow-induced gravity signals will contribute to improving the uncertainty in Saturn's four-layer interior models and to a better understanding of the internal structure and interior composition of Saturn.

Acknowledgements. This work is supported by the Science and Technology Development Fund, Macau SAR (File No. 0005/2019/A1), the Pre-Research Projects on Civil Aerospace Technologies of China National Space Administration (Grant Nos. D020308 and D020303), and the National Natural Science Foundation of China (Grant No. 11761161001).

\section{References}

Anderson, J. D., \& Schubert, G. 2007, Science, 317, 1384

Becker, A., Lorenzen, W., Fortney, J., \& Nettelmann, N. 2014, ApJS, 215, 21

Conrath, B. J., \& Gautier, D. 2000, Icarus, 144, 124

Debras, F., \& Chabrier, G. 2018, A\&A, 609, A97

Galanti, E., Kaspi, Y., Miguel, Y., et al. 2019, Geophys. Res. Lett., 46, 616

Guillot, T. 1999, Planet. Space Sci., 47, 1183

Guillot, T. 2005, Annu. Rev. Earth Planet. Sci., 33, 493

Guillot, T., \& Morel P. 1995, A\&AS, 109, 109

Guillot, T., Miguel, Y., Militzer, B., et al. 2018, Nature, 555, 227

Helled, R., \& Guillot, T. 2013, ApJ, 767, 113

Helled, R., Galanti, E., \& Kaspi, Y. 2015, Nature, 520, 202

Hubbard, W. B. 2013, ApJ, 768, 43

Hubbard, W. B., \& Militzer, B. 2016, ApJ, 820, 80

Hubbard, W. B., Guillot, T., Marley, M. S., et al. 1999, Planet. Space Sci., 47, 1175

Iess, L., Militzer, B., Kaspi, Y., et al. 2019, Science, 364, 2965

Jacobson, R. A., Antresian, P. G., Bordi, J. J., et al. 2006, ApJ, 132, 2520

Kaspi, Y., Guillot, T., Galanti, E., et al. 2017, Geophys. Res. Lett., 44, 5960-8

Kong, D., Zhang, K., \& Schubert, G. 2019, MNRAS, 488, 5633-40

Lindal, G. 1992, AJ, 103, 967

Lorenzen, W., Holst, B., \& Redmer, R. 2011, Phys. Rev. B, 84, 235109

Lyon, S. P., \& Johnson, J. D. 1992, LANL Rep. LA-UR-92-3407 (Los Alamos: LANL)

Mankovich, C., Marley, M. S., Fortney, J. J., \& Movshovitz, N. 2019, ApJ, 871, 1

Militzer, B., \& Hubbard, W. B. 2013, ApJ, 774, 148

Militzer, B., Wahl, S. M., \& Hubbard, W. 2019, ApJ, 879, 78

Miguel, Y., Guillot, T., \& Fayon, L. 2016, A\&A, 596, A114

Miguel, Y., Guillot, T., \& Fayon, L. 2018, A\&A, 618, C2

Morales, M. A., Hamel, S., Caspersen, K., \& Schwegler, E. 2013, Phys. Rev. B, 87, 174105

Nettelmann, N. 2017, A\&A, 606, A139

Nettelmann, N., Fortney, J. J., Moore, K., \& Mankovich, C. 2015, MNRAS, 447, 3422-41

Ni, D. 2018, A\&A, 613, A32

Ni, D. 2019, A\&A, 632, A76

Saumon, D., \& Guillot, T. 2004, ApJ, 609, 1170

Schöttler, M., \& Redmer, R. 2018, Phys. Rev. Lett., 120, 115703

Serenelli, A. M., \& Basu, S. 2010, ApJ, 719, 865

Smith, B. A., Soderblom, L., Batson, R., et al. 1982, Science, 215, 505

Vazan, A., Helled, R., Podolak, M., \& Kovetz, A. 2016, ApJ, 829, 118

Wahl, S. M., Hubbard, W. B., Militzer, B., et al. 2017, Geophys. Res. Lett., 44 4649

Zharkov, V. N., \& Trubitsyn, V. P. 1975, AZh, 52, 599

Zharkov, V. N., \& Trubitsyn, V. P. 1978, Physics of Planetary Interiors(Amsterdam: Elsevier) 


\section{Appendix A: ToF to fifth order compared with the accelerated CMS method}

Here, we examine the accuracy of the ToF to fifth order (ToF5). The comparison with the accelerated CMS method is performed with a similar procedure for Jupiter (Ni 2019). First, we converted the ToF-based density profile as a function of the mean radius $s$ to a density profile as a function of the equatorial radius $r$ using the figure functions $s_{2 i}(s)$. The gravitational harmonics are then recalculated using the accelerated CMS method and the differences between the ToF5-fit and CMS gravitational harmonics are evaluated. Second, the accelerated CMS method is used to fit the rigid-rotation gravitational harmonics instead of ToF5. The resulting gravitational harmonics and model predictions are compared with the ToF5 results. Table A.1 shows the comparison of the ToF5 and accelerated CMS results for two representative models using REOS3b and $\mathrm{MH} 13+\mathrm{SCvH}$. Their offsets are compared with the error bar of the Cassini Grand Finale gravity measurements (Iess et al. 2019) and the flow-induced gravity corrections of Galanti et al. (2019) and Kong et al. (2019). While the ToF5-fit and CMS-fit results show approximately the same $J_{2}$ values, the offsets in $J_{4}-J_{10}$ are smaller than the error bars of Iess et al. (2019) in particular for the higher-order harmonics. Given the uncertainties in the flow-induced gravity corrections (Galanti et al. 2019; Kong et al. 2019), the offsets in $J_{2}-J_{10}$ become more negligible. Besides, one can see that the effect on the heavy element abundances in Saturn's interior is negligible with respect to the uncertainties discussed in this work. With these in mind, the accuracy of the ToF5 method is sufficient to infer Saturn's interior from the Cassini Grand Finale gravity measurements.

Table A.1. Comparison of the ToF5 and accelerated CMS results for two representative models.

\begin{tabular}{|c|c|c|c|c|c|c|c|c|c|}
\hline & Method & $J_{2} \times 10^{6}$ & $J_{4} \times 10^{6}$ & $J_{6} \times 10^{6}$ & $J_{8} \times 10^{6}$ & $J_{10} \times 10^{6}$ & $Z_{1}$ & $Z_{2}$ & $M_{\text {core }}\left(M_{\oplus}\right)$ \\
\hline \multirow{3}{*}{\multicolumn{2}{|c|}{$\begin{array}{c}\text { Error bar }{ }^{(a)} \\
\qquad J_{2 i}{ }^{(b)} \\
\Delta J_{2 i}{ }^{(c)}\end{array}$}} & 0.028 & 0.037 & 0.087 & 0.205 & 0.420 & & & \\
\hline & & 50.54 & 7.5883 & 4.0932 & -5.5055 & 3.6600 & & & \\
\hline & & -3.855 & 3.032 & 2.187 & -5.023 & 3.399 & & & \\
\hline A & ToF5-fit & 16273.43 & -946.7607 & 84.02621 & -9.35601 & 1.20546 & 0.0640 & 0.0247 & 14.89 \\
\hline B & CMS & 16297.04 & -947.9340 & 84.12987 & -9.38624 & 1.20849 & & & \\
\hline A-B & & -23.61 & 1.1733 & -0.10366 & 0.03023 & -0.00303 & & & \\
\hline $\mathrm{C}$ & CMS-fit & 16273.47 & -946.7624 & 84.03046 & -9.37464 & 1.20687 & 0.0639 & 0.0221 & 15.01 \\
\hline $\mathrm{A}-\mathrm{C}$ & Offsets & -0.04 & 0.0017 & -0.00425 & 0.01863 & -0.00141 & 0.0001 & 0.0026 & -0.12 \\
\hline $\mathrm{D}$ & ToF5-fit & 10275.47 & -946.7622 & 84.63584 & -9.48500 & 1.22661 & 0.0508 & 0.0404 & 13.28 \\
\hline $\mathrm{E}$ & CMS & 16293.55 & -947.7614 & 84.72266 & -9.51365 & 1.22936 & & & \\
\hline D-E & & -20.08 & 0.9992 & -0.08682 & 0.02865 & -0.00275 & & & \\
\hline $\mathrm{F}$ & CMS-fit & 16273.44 & -946.7483 & 84.63424 & -9.50304 & 1.22785 & 0.0507 & 0.0383 & 13.38 \\
\hline D-F & Offsets & 0.03 & -0.0139 & 0.00160 & 0.01804 & -0.00124 & 0.0001 & 0.0021 & -0.10 \\
\hline
\end{tabular}

Notes. The top panel shows the uncertainties on Saturn's gravity harmonics. The second panel (A-C) displays the predictions of the interior model using REOS3b. The bottom panel (D-F) displays the predictions of the interior model using MH13+SCvH. ${ }^{(a)}$ Error bars of the Cassini Grand Finale gravity measurements from Iess et al. (2019). ${ }^{(b)}$ Flow-induced gravity corrections of Galanti et al. (2019). ${ }^{(c)}$ Flow-induced gravity corrections of Kong et al. (2019). 